\title{
Dynamique des cavités érosives : étude du collapse des vortex cavitants
}

\author{
François Avellan et Mohamed Farhat \\ Institut de Machines Hydrauliques et de Mécanique des Fluides \\ Ecole Polytechnique Fédérale de Lausanne
}

\section{Introduction}

Bien que de nombreuses études aient été souvent consacrées au comportement dynamique des cavités sphériques, ce type de cavitation n'a que peu d'importance pour le problème de l'érosion des machines hydrauliques. En effet, les conditions d'exploitation correspondant à ce type de cavitation ne sont atteintes que pour les turbines au voisinage du point du meilleur rendement et souvent pour des cotes d'implantation trop hautes par rapport à celles du prototype. En outre le collapse des bulles formées dans les zones de basse pression se produit à la sortie de la roue au sein même de l'écoulement. En revanche, les cavités transitoires qui se forment dans le sillage d'une poche de cavitation attachée à l'arête d'entrée des aubes sont responsables des sévères érosions par cavitation observées dans la plupart des machines hydrauliques.

Ce type de cavités se forme dans une zone de cisaillement intense qui correspond à la région de fermeture de la poche. Les instabilités qui prennent naissance sur la poche provoquent un lâcher périodique de filaments tourbillonnaires en forme de $U$ que nous appellerons des « vortex cavitants». Alors que les bulles de cavitation peuvent imploser loin de la paroi de l'aube, les vortex doivent s'appuyer sur la paroi, en vertu du théorème de Kelvin, et implosent au voisinage de cette dernière.

Afin d'étudier la dynamique des vortex cavitants, nous avons utilisé le générateur de vortex cavitant (CVG) plus connu sous le nom de "veine tourbillon" [1]. En menant une série d'essais comparatifs sur le CVG et sur différentes installations, nous avons montré [2] que les collapses de cavités produites de manière contrôlée et répétée dans le CVG conduisent à une érosion identique à celle rencontrée dans les machines hydrauliques [3] ou sur notre tunnel de cavitation à grande vitesse [4].

L'objet de cette étude est de décrire les mécanismes hydrodynamiques mis en jeu lors de l'effondrement de ces vortex cavitants. Nous décrirons le fonctionnement hydraulique du CVG et l'instrumentation particulière mise en œuvre pour capturer les phénomènes très rapides qui se produisent lors des collapses de ces cavités. Le dépouillement des résultats nous permet alors de mettre en évidence la formation d'ondes de chocs sphériques très violentes au voisinage de la paroi qui provoquent des dégâts dans des alliages aussi résistants que la Stellite [5].

\section{Dynamics of erosive cavities : study relating to the collapse of cavitating vortexes}

Cavitation erosion of hydraulic machines is mainly due to the collapse of transistory cavities which are formed in the wake of leading edge cavities. These cavities, known as "cavitating vortex", have the characteristics of swirling filaments which are formed according to the intense vorticity field generated in the closing zone of these pockets. With a view to analyzing the dynamics of cavitating vortex, we have used a cavitation vortex generator which allows for producing repeated and fully controlled collapses of this type of cavity. By means of a display system via a high-speed camera (1 milliom images per second) and a simultaneous follow-up of the wall pressure and vapor phase, we have established that these collapses generate waves with extremely violent impacts (10 to $20 \mathrm{Kbars}$ ) and which have initially provoked the damage observed. 


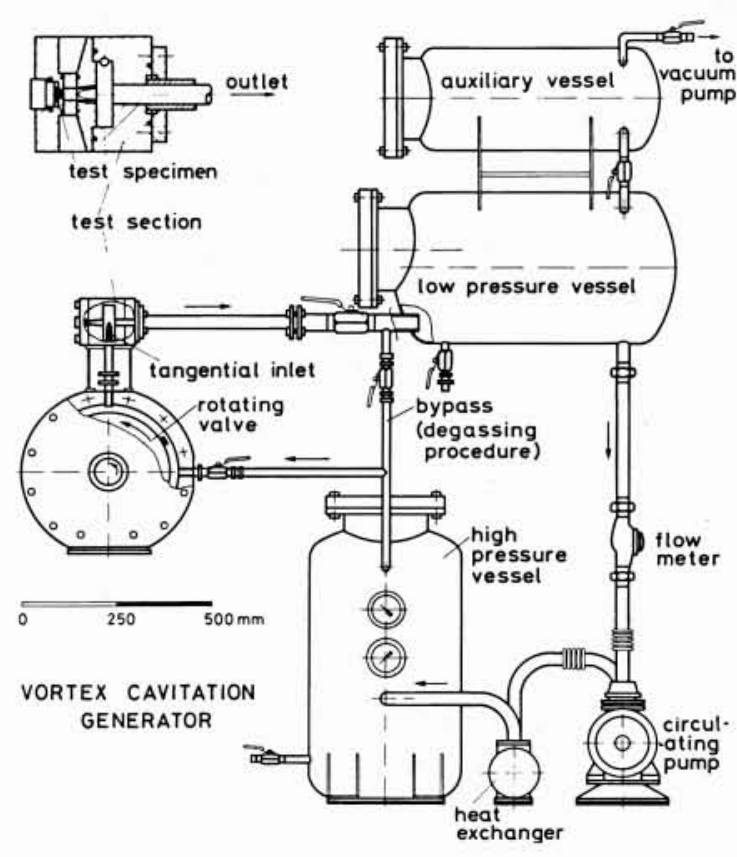

1. Schéma du générateur de vortex cavitants.

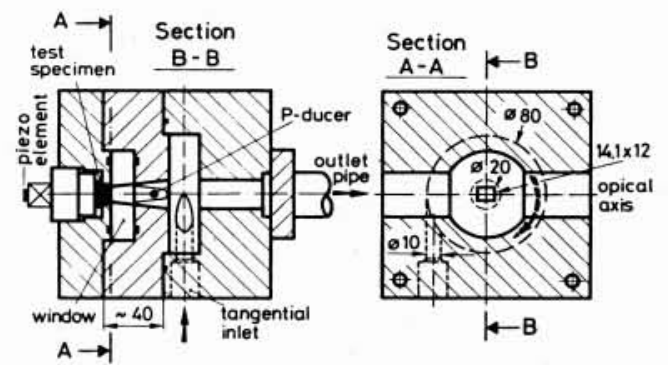

2. Vue de côté et de dessus de la veine d'essai. (Toutes les dimensions sont en mm.)
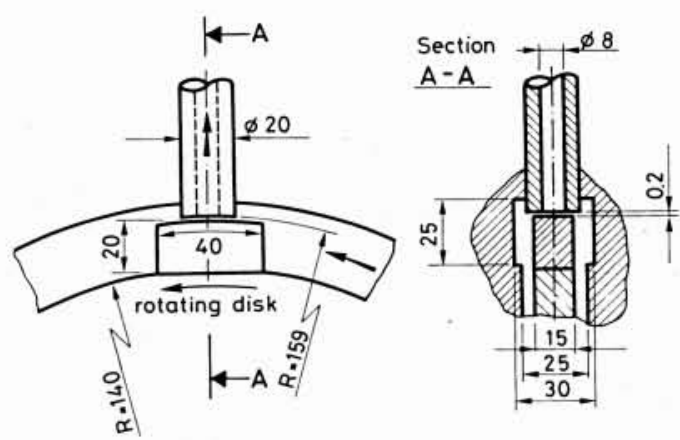

3. Schéma de la vanne rotative. (Toutes les dimensions sont en mm.)

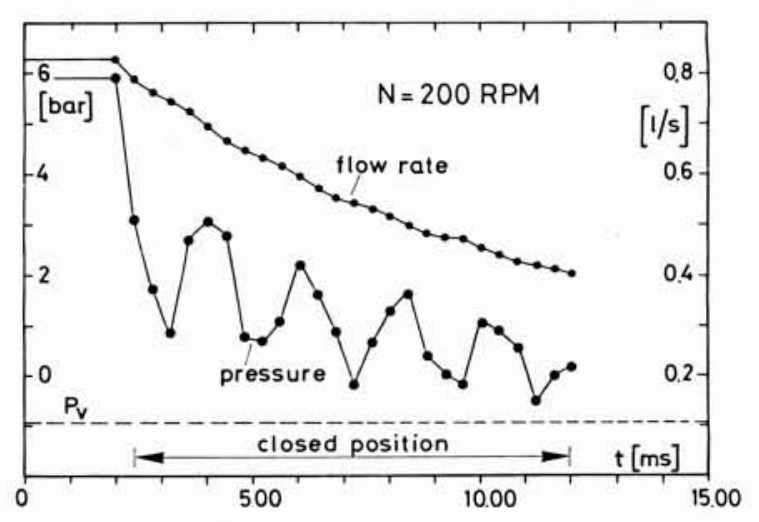

4. Evolution du débit et de la pression dans la veine lors de la fermeture de la vanne.

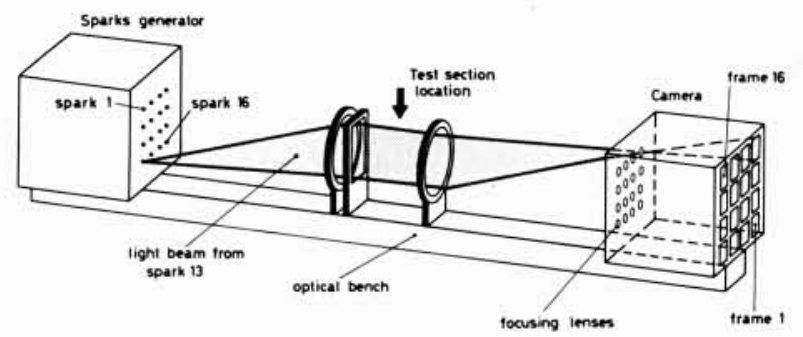

5. Schéma de principe de la caméra CRANZ SCHARDIN.

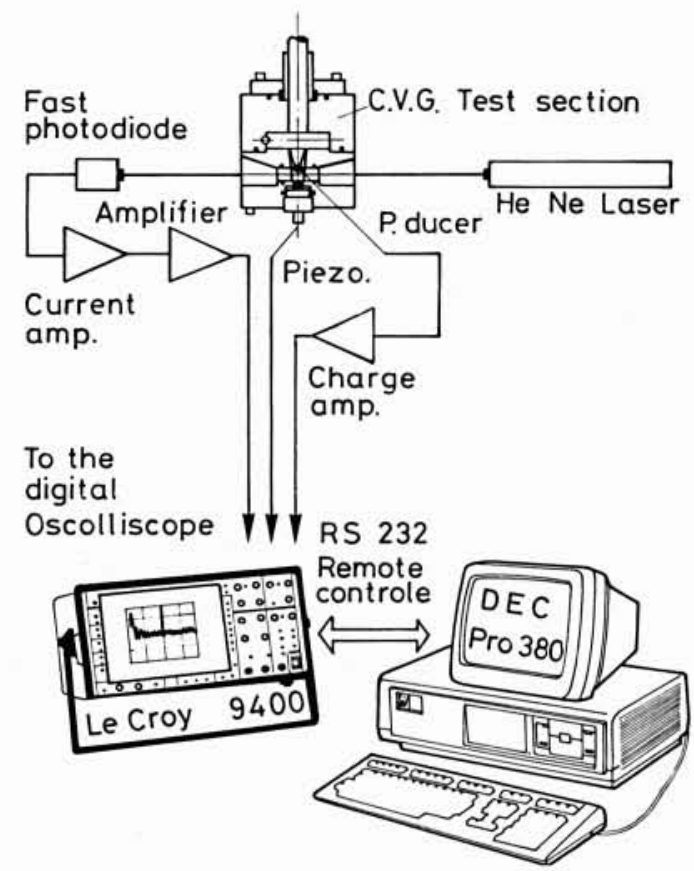

6. Principe de détection de la phase vapeur et le système d'acquisition des signaux. 


\section{Moyens expérimentaux}

\subsection{Le générateur de vortex cavitant}

\section{Description}

Il s'agit d'un circuit hydraulique fermé comprenant une veine d'essai, où des essais d'érosion et de visualisation peuvent être conduits, une vanne rotative, un ensemble de plusieurs réservoirs et une pompe de circulation (fig. I) La pompe aspire l'eau dans le réservoir à pression atmosphérique et la refoule à travers un échangeur de chaleur dans le réservoir de haute pression. Ce dernier, qui possède un volume total de 601 , joue le rôle d'une chambre tampon capable d'atténuer les variations brusques de pression dans le circuit. Le débit maximum atteint est de $3 \mathrm{~m}^{3} / \mathrm{h}$, ce qui correspond à une pression amont de 9,5 bar.

La veine d'essai est constituée d'une chambre cylindrique de $80 \mathrm{~mm}$ de diamètre et de $20 \mathrm{~mm}$ de largeur, terminée d'un côté par un cône tronqué d'une longueur de $40 \mathrm{~mm}$ réalisant un changement de section circulaire à rectangulaire et de l'autre par un conduit de $24 \mathrm{~mm}$ de diamètre et $850 \mathrm{~mm}$ de longueur débouchant dans le réservoir aval. L'entrée du fluide dans la veine d'essai se fait de manière tangentielle par un conduit de $10 \mathrm{~mm}$ de diamètre (fig. 2).

La vanne rotative est constituée d'un disque de $140 \mathrm{~mm}$ de diamètre entraîné en rotation par un moteur à courant continu dont la vitesse est variable. L'obturateur proprement dit, de $19 \mathrm{~mm}$ de hauteur et de $40 \mathrm{~mm}$ de longueur, est fixé sur la périphérie du disque (fig. 3). L'entrefer entre l'obturateur et le conduit d'amenée est de $0,2 \mathrm{~mm}$.

\section{Fonctionnement}

Le principe de la veine tourbillon est de produire une série d'ondes de détente et de compression telles qu'une génération de cavité de vapeur suivie d'un collapse ait lieu à chaque rotation de la vanne. Pendant la phase d'ouverture de la vanne qui représente $96 \%$ de la durée d'un cycle, et à cause de l'entrée tangentielle, un écoulement du type vortex s'établit dans la veine et la branche aval. Contrairement à un tourbillon libre, les effets visqueux limitent la vitesse dans le voisinage de l'axe du vortex. Dans cette zone, l'écoulement est du type à rotation solide.

La fermeture de la vanne provoque un coup de bélier dans l'installation : une onde de détente se propage alors dans la veine et la conduite aval. Celle-ci donne naissance à un noyau de vapeur centré sur l'axe du vortex qui tend à s'étendre sur tout le rayon. La durée de fermeture de la vanne qui dépend de la vitesse de rotation limite l'expansion du noyau de vapeur.

Un calcul de coup de bélier, conduit pour un débit de $3 \mathrm{~m}^{3} / \mathrm{h}$ et une vitesse de rotation de la vanne de $200 \mathrm{t} / \mathrm{min}$, permet de tracer l'évolution du débit et de la pression dans la veine (fig. 4). Il est à noter que la vaporisation de l'eau a lieu au niveau de la vanne dès la fermeture totale de cette dernière. Par ailleurs, la présence de gaz dissous provoque la formation d'une poche de vapeur stationnaire quelle que soit la position de la vanne, ce qui atténue l'intensité des collapses. Aussi, avant chaque expérience, on procède à un dégazage de l'eau au moyen d'une pompe à vide reliée au réservoir aval. Le taux de gaz dissous, contrôlé par une sonde d'oxygène, est maintenu en dessous de $1 \mathrm{ppm}$.

\subsection{Caméra ultra-rapide}

Afin de visualiser les mécanismes de formation et du collapse de la cavité de vapeur dans la veine, nous avons utilisé un système de prise de photo ultra-rapide de type CRANZ SCHARDIN et qui, à l'origine, a été développé à l'Institut Saint-Louis [6]. Ce système comprend une batterie de 16 sources flashes quasi ponctuelles et coplanaires et d'une caméra dotée de 16 objectifs ainsi qu'un arrangement optique faisant correspondre à chacune des sources de lumière un objectif de la caméra de telle sorte que l'on obtienne sur la surface sensible 16 images indépendantes de $14 \mathrm{~mm} \times 32 \mathrm{~mm}$. Chacun des flashes est constitué de deux électrodes et l'éclair provient d'une décharge dans l'air de 2,4 Joules d'un condensateur de $47 \mathrm{nF}$ alimenté à $10 \mathrm{KV}$. La durée de l'éclair, mesurée au $1 / 3$ du pic, est de $500 \mathrm{~ns}$. Une unité de contrôle permet de choisir la fréquence de déclenchement des flashes entre $10 \mathrm{kHz}$ à $10 \mathrm{MHz}$ avec une résolution de $0,1 \mu \mathrm{s}$.

L'arrangement optique qui permet d'obtenir un éclairage par transmission en lumière parallèle de la veine, comprend deux lentilles convergentes identiques de $1600 \mathrm{~mm}$ de longueur focale, placées de part et d'autre de la veine d'essai. Les sources de lumière sont placées dans le plan focal objet de la première lentille alors que les objectifs, de $380 \mathrm{~mm}$ de longueur focale et de $11,8 \mathrm{~mm}$ de diamètre chacun, sont disposés dans le plan focal image de la deuxième lentille (fig. 5). Afin d'améliorer le rendement lumineux, les faces transparentes de la veine ont été réalisées en verre de haute qualité optique avec un traitement anti-reflet. Le déclenchement de la caméra se fait à partir de la fermeture de la vanne avec un retard programmable.

\subsection{Détection de la phase vapeur}

Afin de contrôler la présence de la phase vapeur de manière continue, nous avons illuminé la veine par un faisceau laser, $\mathrm{He} \mathrm{Ne}$, d'une puissance de $2 \mathrm{~mW}$. Le faisceau transmis est recueilli par une photodiode ultra-rapide, EG \& G type FND100, dont le temps de montée est de $1 \mathrm{~ns}$ et la bande passante de $350 \mathrm{MHz}$ (fig. 6).

\subsection{Mesure de pression et d'accélération}

Afin de suivre l'évolution de la pression en paroi au niveau de la veine, nous avons utilisé un capteur à quartz de type KISTLER 603 B. Le temps de montée est de $1 \mu \mathrm{s}$, la fréquence propre est de $400 \mathrm{kHz}$ et le domaine de mesure s'étend de 0 à 200 bar. Le signal ainsi obtenu est amplifié et converti à travers l'amplificateur de charge VIBROMETRE TA 3/D (bande passante de $300 \mathrm{kHz}$ ). 

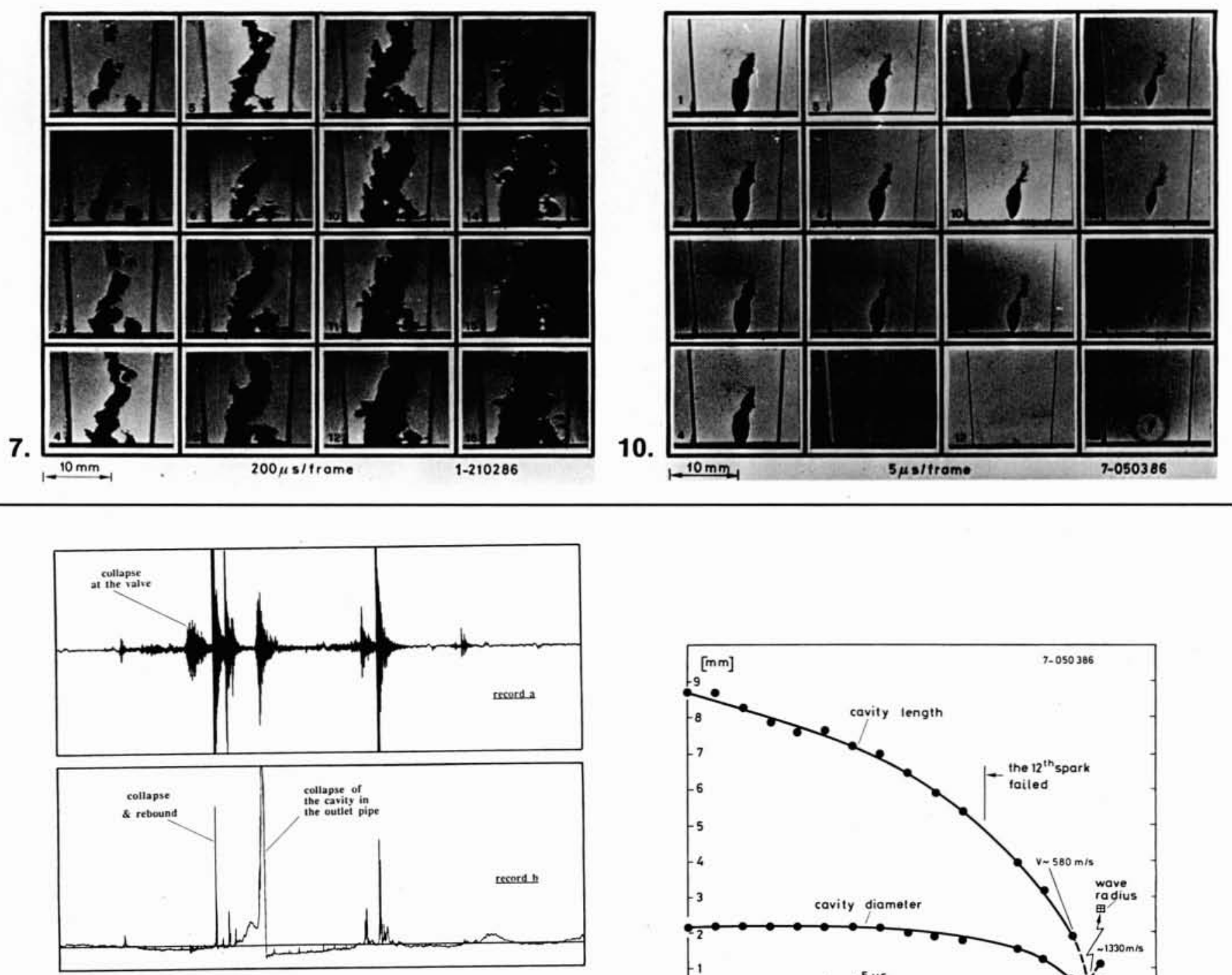

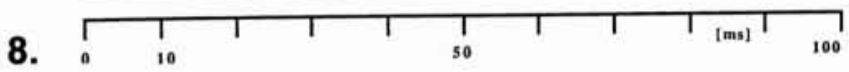
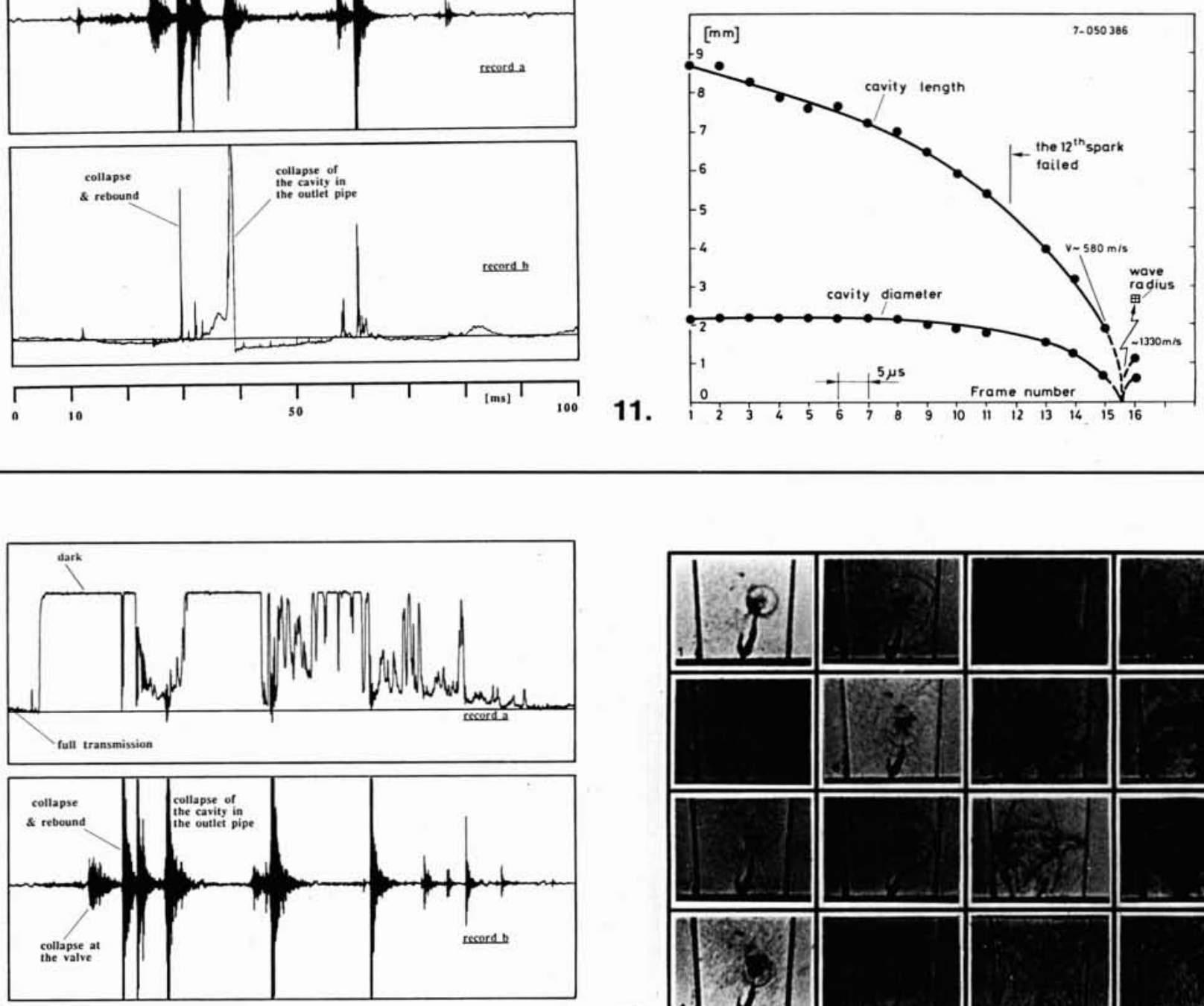

9.
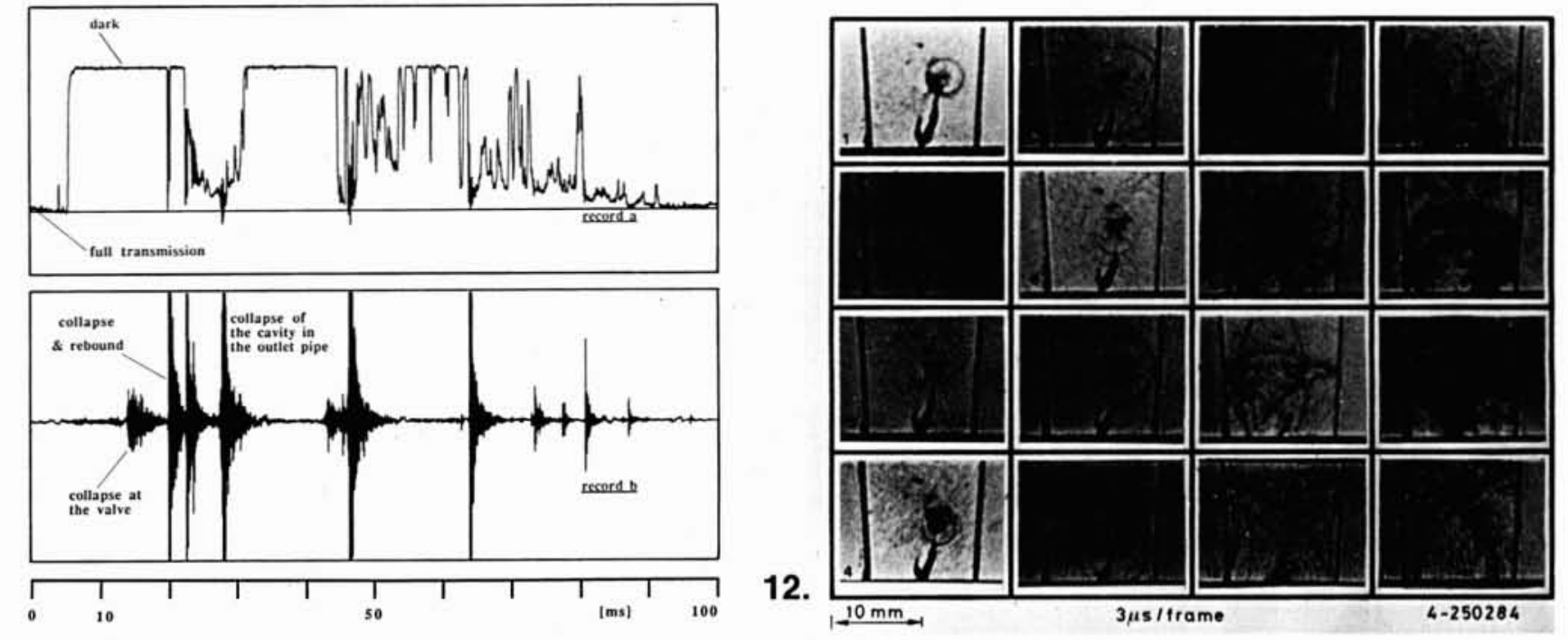
7. Naissance et explosion du vortex cavitant. Période d'éclairs : $200 \mu$ s, débit moyen : $3 \mathrm{~m}^{3} / \mathrm{h}$, vitesse de rotation de la vanne: $200 \mathrm{t} / \mathrm{mn}$.

8. Enregistrement simultané des signaux de pression (a) et d'accélération $(b)$. Débit moyen : $3 \mathrm{~m}^{3} / \mathrm{h}$, vitesse de rotation de la vanne : $200 \mathrm{t} / \mathrm{mn}$

9. Enregistrement du signal de la photodiode (a) et celui de l'accéléromètre $(b)$. Débit moyen : $3 \mathrm{~m}^{3} / \mathrm{h}$, vitesse de rotation de la vanne : $200 \mathrm{t} / \mathrm{mn}$

10. Stade final du collapse du vortex cavitant. Période d'éclairs: $5 \mu \mathrm{s}$, débit moyen : $3 \mathrm{~m}^{3} / \mathrm{h}$, vitesse de rotation de la vanne: $200 \mathrm{t} / \mathrm{mn}$.

11. Evolution de la longueur et du diamètre de la cavité de la séquence de la figure 10.

12. Stade final du collapse du vortex cavitant. Période d'éclairs : $3 \mu \mathrm{s}$, débit moyen : $3 \mathrm{~m}^{3} / \mathrm{h}$, vitesse de rotation de la vanne: $200 \mathrm{t} / \mathrm{mn}$.

En outre, un accéléromètre à effet piézo-électrique, PHILIPS PXE 41, a été placé derrière l'échantillon afin de rendre compte des vibrations générées par le phénomène du collapse au niveau de la veine.

Tout comme celle du signal issu de la photodiode, l'acquisition des signaux de pression et d'accélération se fait au moyen de l'oscilloscope digital LeCroy DO9400. La fréquence d'acquisition est de $120 \mathrm{MHz}$. La longueur d'un enregistrement est de 32000 points. Le transfert des signaux depuis les mémoires de l'oscilloscope à l'ordinateur, DEC Pro 380, est rendu possible par un logiciel développé à cet effet.

\section{Résultats}

Les essais présentés dans ce papier ont tous été réalisés pour une vitesse de rotation de la vanne de $200 \mathrm{t} / \mathrm{min}$ et un débit moyen de $3 \mathrm{~m}^{3} / \mathrm{h}(0,8 \mathrm{l} / \mathrm{s})$.

La séquence de photos synchronisée avec l'acquisition des signaux de pression et d'accélération d'une part, ainsi que l'enregistrement simultané de chacun de ces signaux avec le signal photoélectrique d'autre part, permettent de reconstituer l'histoire de la poche de vapeur dans les phases d'explosion et de collapse.

La séquence de photos de la figure 7 a été prise $7 \mathrm{~ms}$ après le début de la fermeture de la vanne et la durée entre deux éclairs est de $200 \mu$ s. Elle montre que l'onde de détente provoque tout d'abord la formation de petites cavités dans la veine et le conduit aval et que, par la suite, ces cavités grossissent et se réunissent pour former un noyau de vapeur centré sur l'axe du vortex. Tant que la vanne reste fermée, ce noyau s'étend dans la direction radiale avec une vitesse d'expansion estimée à $1,7 \mathrm{~m} / \mathrm{s}$. Durant cette phase, le capteur de pression indique bien une baisse de la pression dans la veine alors que l'accéléromètre demeure jusque-là indifférent (fig. 8).
L'ouverture de la vanne génère une onde de compression qui, comme on le conçoit, fait imploser immédiatement la poche de vapeur dans le conduit amont. Le choc qui en résulte est parfaitement ressenti par l'accéléromètre. Dans la veine, l'expansion du noyau de vapeur prend fin et après $6 \mathrm{~ms}$ environ ce dernier disparaît complètement. Il en résulte une génération d'ondes de choc très violentes ressenties simultanément par le capteur de pression $\left(1^{\text {er- }}\right.$ pic) et par l'accéléromètre, suivies d'un rebond puis d'un deuxième collapse comme le montre le signal photoélectrique (fig. 9).

D'autre part, le pic observé sur le signal de pression, beaucoup plus violent que le premier, est d'autant plus surprenant qu'à cet instant même, le signal photoélectrique indique une absence totale de la vapeur immédiatement avant et après. Ceci a été confirmé par un balayage systématique de cette zone avec la caméra ultra-rapide. Le pic en question est attribué au collapse du noyau de vapeur au niveau de la branche aval. En effet, l'arrivée de l'onde de compression au droit de la veine provoque une scission du barreau de vapeur en deux parties : la première, située dans la veine, la seconde, de taille plus importante, dans le conduit aval. La différence de tailles entre ces deux cavités explique pourquoi c'est, d'abord, la première qui implose.

On assiste ensuite à un développement d'ondes de compression et de détente très amorties qui se propagent entre le réservoir aval et la veine d'essai. Il en résulte une successions quasi-périodique de collapses et de rebonds d'intensité décroissante et parfaitement visibles sur les signaux de pression et d'accélération ainsi que sur le signal de la photodiode (fig. $8 \& 9$ ). Les séquences de photos représentées dans les figures 10 et 12 , correspondent au stade final du collapse. Toutes les deux ont été prises avec un retard de $24 \mathrm{~ms}$ par rapport à la fermeture de la vanne. La séquence de la figure 10 a été effectuée avec une durée inter-éclairs de $5 \mu \mathrm{s}$. On y observe un effondrement de plus en plus accéléré de la poche de vapeur suivie d'une onde sphérique générée entre la $15^{\mathrm{c}}$ et la $16^{\mathrm{e}}$ image qui est visible sur cette dernière. Nous avons reporté sur la figure 11 l'évolution du diamètre et de la longueur de la poche. On en déduit que l'effondrement selon l'axe est plus prononcé que selon le diamètre. Les accélérations axiale et radiale moyennes valent respectivement $2,4 \mathrm{~m} / \mathrm{s}_{2}$ et $0,98 \mathrm{~m} / \mathrm{s}^{2}$.

L'extrapolation des courbes de la figure 11 , en supposant que le mouvement axial et le mouvement radial sont uniformément accélérés, nous donne un instant de collapse égal à $2,4 \mu$ s ou $4,4 \mu$ s après la $15^{\mathrm{e}}$ image, suivant que l'on considère la longueur ou le diamètre. En prenant un temps de collapse moyen de $3,2 \mu \mathrm{s}$, on trouve une vitesse de rebond de $690 \mathrm{~m} / \mathrm{s}$ selon l'axe et de $375 \mathrm{~m} / \mathrm{s}$ selon le rayon. En outre, la mesure du rayon de l'onde sur la dernière photo entraine une vitesse de propagation de $1660 \mathrm{~m} / \mathrm{s}$. Dans ce cas et en utilisant l'équation du choc normal, on aboutit à une différence de pression de part et d'autre du front d'onde de 6,2 kbar en supposant la vitesse du fluide égale à la vitesse radiale de la cavité et de $11,4 \mathrm{kbar}$ pour une vitesse du fluide égale à la vitesse axiale de la cavité.

La séquence de la figure 12 a été réalisée avec une période inter-éclair de $3 \mu$ s afin de pouvoir suivre l'évo- 
lution des ondes de choc et leur interaction avec la cavité de vapeur. On observe alors l'existence d'une cavité principale attachée à la paroi ainsi que plusieurs autres cavités secondaires isolées qui collapsent et réapparaissent plusieurs fois. Un traitement informatique de ces photos a été mis au point. Il consiste à digitaliser les fronts d'ondes et à estimer leur rayon au sens des moindres carrés. Le facteur de grandissement a été évalué en tenant compte de la distorsion optique.

Nous avons représenté sur la figure 13, l'évolution de la longueur de la cavité principale, les rayons des ondes de choc, auxquels nous avons ajouté la cote du centre d'émission, ainsi que les vitesses moyennes de propagation de ces ondes. Ces dernières ont été soulignées pour les ondes réfléchies. On remarque que l'effondrement de la cavité principale est accéléré à chaque génération d'ondes due au collapse de la cavité voisine (fig. 13). L'implosion de la cavité principale a lieu entre la $12^{\mathrm{e}}$ et la $13^{\mathrm{e}}$ photo à une distance de 1,6 mm de la paroi. Les ondes de choc ainsi que le rebond qui en résultent sont visibles sur les photos 14 , 15 et 16 (fig. 12).

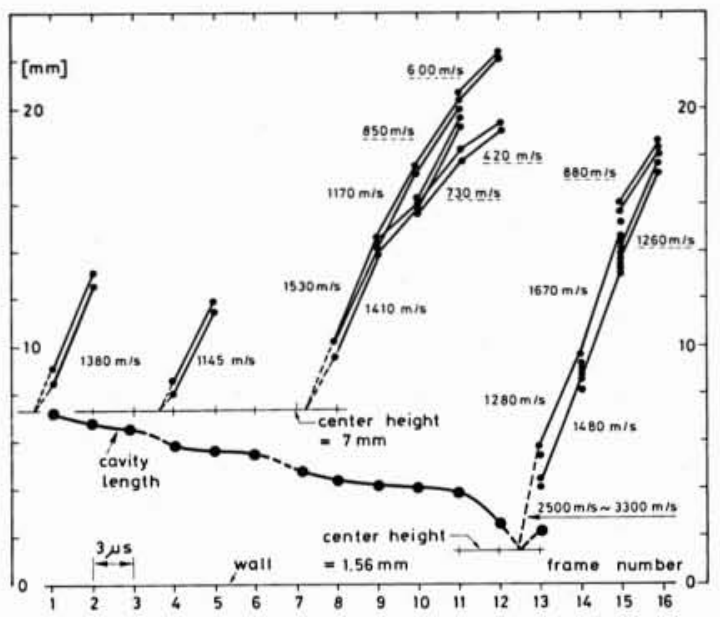

13. Evolution de la longueur de la cavité principale et des rayons d'ondes de choc correspondants à la séquence de la figure 11 .

L'extrapolation de la courbe représentative de longueur de cavité, basée sur l'hypothèse d'accélération constante, donne un instant de collapse égal à $1,8 \mu$ s après la $12^{\mathrm{C}}$ photo. On en déduit une vitesse de rebond égale à $690 \mathrm{~m} / \mathrm{s}$ et une célérité d'onde égale à $3300 \mathrm{~m} / \mathrm{s}$. La différence de pression à travers le front d'onde calculée dans l'hypothèse du choc normal est alors de 22,7 kbar. D'autre part, le nombre de Mach, calculé pour cette vitesse de propagation de l'onde et en supposant la célérité du son égale à $1490 \mathrm{~m} / \mathrm{s}$, est égal à 2,23 . En adoptant l'équation d'état de TAIT pour l'eau, on déduit une différence de pression de part et d'autre du front d'onde égale à $32,6 \mathrm{kbar}$. Ces valeurs différentes de pression indiquent que l'on a certainement surévalué le temps.

$\mathrm{Si}$ on reprend l'estimation de l'instant de collapse en supposant que la cavité évolue de la même manière avant et après le choc, on trouve un temps de 1,7 $\mu \mathrm{s}$. Dans ce cas, la vitesse du rebond est de $640 \mathrm{~m} / \mathrm{s}$ et la célérité d'onde est de $3050 \mathrm{~m} / \mathrm{s}$. Ce qui donne une différence de pression, dans l'hypothèse du choc normal, égale à 19,5 kbar. Le nombre de Mach correspondant est de 2,06, l'équation de TAIT donnant alors une différence de pression de $25,7 \mathrm{kbar}$. II semble que cette estimation de 1,7 $\mu$ s soit plus réaliste, car la valeur obtenue à partir de l'équation de TAIT se rapproche de la valeur obtenue à partir de l'hypothèse du choc normal.

Il ressort néanmoins de ces derniers calculs que la détermination de la pression est très sensible à la détermination de l'instant du collapse. Toutefois, l'ordre de grandeur des surpressions calculées ci-dessus demeure réaliste et explique comment la cavitation peut provoquer des déformations plastiques sur n'importe quel matériau industriel.

\section{Conclusion et perspectives}

Le CVG nous permet d'étudier à un niveau microscopique les processus dynamiques mis en jeu lors de l'effondrement des vortex cavitants.

Le suivi simultané de la présence de la phase vapeur, de la pression et de l'évolution des cavités obtenue par caméra ultra-rapide nous permet de mettre en évidence la formation d'ondes de choc sphériques très violentes à l'instant du collapse. En outre nous n'avons pas pu observer d'instabilités d'interface qui auraient pu engendrer un micro-jet comme pour le cas des cavités sphériques. Au contraire, le niveau de surpression créé par les ondes de choc, estimé entre 10 et $20 \mathrm{kbar}$, permet d'expliquer à lui seul que ce type de cavitation puisse endommager n'importe quel alliage industriel.

La sensibilité du calcul de pression à l'estimation précise de l'instant du collapse a mis en évidence la nécessité d'augmenter la cadence de prise de vues. Or, l'examen d'un très grand nombre d'enregistrements de signaux de pression et d'accélération montre que l'instant du collapse compté à partir du début de l'ouverture de la vanne n'est pas reproductible d'un cycle à l'autre. L'incertitude qui en découle sur le déclenchement de la caméra est estimée à une milliseconde. Pour résoudre cette difficulté, nous avons développé un système de déclenchement à partir de l'oscilloscope numérique qui nous permettra très prochainement de déclencher la caméra à partir du signal de la pression ou de la photodiode et par suite d'accroître le taux de réussite au niveau des séquences photo.

Par ailleurs le traitement d'un très grand nombre de photos nous permettra, entre autres, d'établir les bases pour une modélisation numérique du collapse des vortex cavitants.

Adresse des auteurs

Messieurs F. Avellan et $M$. Farhat

IMFL, Avenue de Cour, 33

CH 1007 Lausanne (Suisse)

Tél. : (19) 4121472524 


\section{Références}

[1] Lecoffre, Y., Marcoz, J. \& Valibouse, B., 1981. - Proc. ASME Fluids Engineering Conference, Boulder, June, 1981.

[2] Karimi, A. \& Avellan, F., 1986. - WEAR, ed. Elsevier, vol. 113, no 3, pp. 305-322, December 1986.

[3] Avellan, F. \& Karimi, A., 1987. - Proc. 7th International Conference on Erosion by Liquid and Solid Impact, September 1987, pp. $25-1,25-8$
[4] Henchoz, A., 1987. - Proc. of Conference on High Speed Photography and Photonics, Tokyo, August 1987.

[5] Avellan, F. \& Henry, P., 1987. - Proc, EPRI POWER Plant Pumps Symposium, ed. Electric Power Research Institute, New Orleans, March 1987, pp. 1-22.

[6] Avellan, F., Henry, P. \& Ryhming, L., 1987. - Proc. ASME Int. Winter Annual Meeting, Boston, December 1987.
M. CHIEM: Je félicite l'équipe de Lausanne, car la technique Cranz-Schardin n'est pas simple à mettre en œuvre.

M. HEUZÉ : Pourquoi avez-vous changé la forme géométrique de la section de veine en passant d'une section circulaire à une section rectangulaire?

M. FARHAT: C'est pour pouvoir réaliser les visualisations par ombroscopie. On a par ailleurs remarqué que la géométrie prismatique conduisait à une meilleure stabilité des vortex. Les dégâts sont plus centrés et plus importants.

M. GRISON: Concernant les mesures de pression et d'accélération, on constate que la corrélation temporelle des deux signaux est bonne, alors que la corrélation en amplitude ne l'est pas. Comment expliquez-vous cela?

M. FARHAT: L'amplitude en temps que telle ne veut rien dire. Il faut tenir compte des différents gains des amplificateurs et de la réponse en fréquence des capteurs. Par ailleurs, les capteurs de pression n'ont pas été utilisés pour mesurer la pression elle-même, mais pour détecter les événements qui se produisent.

M. GRISON : Sans attacher d'importance à la valeur absolue, étant donné que les deux enregistrements sont simultanés, la corrélation relative devrait être bonne.

M. AVELLAN : Certaines vibrations sont dues aux oscillations en masse et aux collapses produits dans le conduit aval. Elles sont détectées par l'accéléromètre. Au contraire, le capteur de pression enregistre ce qui se passe dans la veine elle-même. C'est un capteur KISTLER 603B, isolé des accélérations. On avait essayé de se placer à la source du phénomène, mais nous avions des résultats identiques.

Quant au deuxième pic de pression, que nous n'arrivions pas à interpréter, il provient de la colonne de vapeur présente dans le conduit aval. L'implosion de cette colonne engendre une grande variation de pression, insuffisante cependant pour endommager le matériau.

A la limite, si on plaçait un capteur de pression sous l'impact, on ne verrait rien, car il serait détruit. Toute l'énergie passerait dans cette destruction et non pour engendrer un signal.

M. CHIEM : Pourquoi dites-vous que la vitesse de rebond est trop faible?

M. AVELLAN : On a une onde réfléchie dont la vitesse devrait être au moins de $1400 \mathrm{~m} / \mathrm{s}$ en eau dégazée d'après ce que je sais des fluides incompressibles. On trouve jusqu'à $600 \mathrm{~m} / \mathrm{s}$, ce qui ne correspond pas à des valeurs communes.

M. CHIEM : Il peut y avoir un problème d'impédance mécanique ou de relation entre les densités du matériau et du liquide. L'atténuation de l'onde réfléchie dépend de l'emplacement où arrive l'onde incidente.

M. AVELLAN: Il s'agissait de propagation élastique dans le fluide lui-même et ces phénomènes liés au matériau des parois ne peuvent pas intervenir.
K. KARIMI : La pression hydrostatique dans la veine tourbillon influence-t-elle les ondes de choc?

M. AVELLAN: Pas à ces valeurs.

M. BARRAND : Permettez-moi de suggérer une piste. Des essais ont été faits au Laboratoire de Mécanique des Fluides de l'INSA de Lyon: des impacts d'ondes de choc sur des obstacles rigides ou déformables effectués en cuve hydraulique à la pression atmospherique ont mis en evidence dans certains cas la creation d' " une cavitation en $\mu$ bulles locales ". Ceci pourrait expliquer, peut-être, une diminution de la célérité des ondes.

M. AVELLAN : Je penche vers cette suggestion: on a souvent observé des microbulles fines autour de la cavité principale, au rebond. Notre résolution n'étant que de $1 / 100 \mathrm{de} \mathrm{mm}$, on ne peut cependant pas voir ces microbulles.

M. LECOFFrE: Je félicite MM. FARHAT et Avellan pour la qualité de leurs expériences, et, en particulier pour leur visualisation d'ondes de choc. Nous avions essayé de le faire à Neyrtec il y a quelques années sans y réussir.

Je voudrais également faire remarquer que les conditions d'utilisation par Lausanne de la veine tourbillon sont des conditions extrêmes, les impacts produits étant sans doute plus violents que ceux que l'on rencontre dans les machines industrielles. Lors du développement de cette veine, nous nous sommes, pour notre part contentes de chocs moins violents, les réglages de la veine étant ceux qu'utilise M. Heuzé. Selon le réglage, la production éventuelle de microbulles peut être différente.

M. AVELLAN: Ce que l'on a cherché à faire, c'est d'augmenter la vitesse d'érosion. Les études hydrodynamiques sont venues seulement après.

Il était à notre avis plus fondamental de montrer qu'on attaque les matériaux les plus durs et même la stellite dont la limite de rupture est de l'ordre de 2000 méga pascal.

C'est une preuve de ce que le mécanisme peut engendrer comme type de dégâts. On peut rencontrer toutes sortes de mécanismes, la bulle spécifique qui collapse, le microjet, l'onde de choc, qui font des trous dans du nougat ou dans du beurre, et aussi des phénomènes plus violents qui provoquent des dégâts dans la stellite.

M. HEUZÉ: Attribuez-vous l'endommagement au collapse initial ou au rebond?

M. FARHAT: Je crois que le responsable est le premier collapse. La suite est de moindre importance.

M. AVELLAN : On parle de rendement pour la veine tourbillon, mais c'est en fait un rendement à l'envers, caractérisé par un nombre de trous par tour.

M. MAAMOURI: Je crois que les dégâts sont dus aux ondes de choc. Nous avons obtenu jusqu'à $150 \%$ d'efficacité avec des matériaux mous. Il y a, de toute façon, une limite liée à la résistance des matériaux. 


\section{OUVRAGES ÉDITÉS PAR LA SOCIÉTÉ HYDROTECHNIQUE DE FRANCE \\ 119, rue de Grenelle - 75007 PARIS. Tél. (1) 47.05.13.37 \\ (Les prix indiqués sont T.T.C.)}

\section{COMPTES RENDUS DES « JOURNÉES DE L'HYDRAULIQUE »}

$\mathrm{XI}^{\circ} \quad$ Utilisation des ressources en eau d'un bassin dans le cadre de l'aménagement du territoire (Paris, 1970) 720 p

$\mathrm{XII} \quad$ Hydrotechnique des liquides industriels ( $\mathrm{Pa}$ ris, 1972) $632 p$

XIVe La mécanique des fluides et l'environnement (Paris, 1976) $630 \mathrm{p}$

$X V^{*} \quad$ L'hydrotechnique au service d'une politique de I'eau (Toulouse, 1978) $650 \mathrm{p}$
XVI $\quad$ Rejet de chaleur à l'atmosphère - Gestion des calories et hydraulique associée (Paris, 1980) $690 p$

$500 \mathrm{~F}$

XVII' L'assainissement de demain (Nantes, 1982) $720 \mathrm{p}$

XVIII L'hydraulique et la maitrise du littoral (Marseille, 1984).

$500 \mathrm{~F}$

$\mathrm{XIX}^{\circ} \quad$ Impact des activités humaines sur les eaux continentales (Paris, 1986)

\section{AUTRES DOCUMENTS}

Cahier des charges type pour la fourniture et le montage des conduites forcées en acier et de leurs accessoires (Edition : octobre 1972) $66 \mathrm{p} . . . .$.

Données techniques. Annexes au Cahier des charges pour la fourniture et le montage des conduites forcées en acier et de leurs accessoires (Edition : décembre 1968) $142 p$.

Cahiers des charges type pour la fourniture de turbines hydrauliques et matériels associés :

Fascicule1: Spécifications concernant l'ensemble des turbines Francis, Pelton et Kaplan et spécifications particulières propres aux turbines Francis (Edition : avril 1972) $126 p$

Fascicule 2: Spécifications particulières propres aux turbines Pelton (Edition : juin 1972) $30 p$............

Fascicule 3: Spécifications particulières propres aux turbines Kaplan (Edition : janvier 1973) 26 p..

Fascicule 4: Spécifications complètes concernant les groupes du type Bulbe (Edition: décembre 1974) $120 \mathrm{p}$

Traduction en anglais des mêmes fascicules

Fascicule 1: 152 p

Fascicule 2: $31 \mathrm{p}$

Fascicule 3: $36 \mathrm{p}$

Fascicule 4 : $135 \mathrm{p}$

Vibrations de structures dans un écoulement sous l'effet du détachement tourbillonnaire de sillage (1981) $202 \mathrm{p}$

Vibrations des structures en présence d'un milieu fluide sans écoulement (1982) $104 p$

Précipitations atmosphériques (1984)
Guide pour la conception, la réalisation, la mise en service et l'exploitation des petits aménage ments hydro-électriques (1985) $250 \mathrm{p}$

Etude des fonctionnements à débit partiel des turbomachines à faible variation volumique du fluide - Particularités propres aux turbines et aux pompes-turbines (Document élaboré par le sous-groupe de travail 1. d: H. Amblard, G. Borciani, P. Guiton, P. Henry, G. Martin et R. Thalmann)

Comptes-rendus du XIV congrès de I'Association internationale de recherches hydrauliques (Paris, 1971) : La recherche hydraulique et son impact sur l'environnement

Volume 1 - Problèmes de transfert dans les écoulements liquides

Volume 2 - Forces hydrodynamiques en régime non-stationnaire

Volume 3 - Déformation des lits alluvionnaires composés de matériaux à granulométrie étendue

Volume 4 - Sédimentation dans les estuaires et les embouchures

Volume 5 - Compte-rendu des cinq Séminaires

Volume 6 - Discussions des allocutions

Les 6 volumes brochés (Frais d'envoi en sus)

Comptes-rendus du "Symposium AIRH/SHF de Grenoble (1976): Ecoulements diphasiques et cavitation dans les systèmes de production d'énergie 》"

Les deux volumes brochés

(Franco de port et d'emballage)

DERNIERS OUVRAGES PARUS (1987)

Small scale hydropower schemes - 262 p. $475 \mathrm{~F}$

Guide de prévision des crues -750 p. $600 \mathrm{~F}$ 